\title{
SISTEMA DE AHORRO ENERGÉTICO BASADO EN COLECTORES SOLARES PARA DOTAR DE AGUA CALIENTE SANITARIA (ACS) A UNA VIVIENDA SOCIAL DE 2 DORMITORIOS EN LA CIUDAD DE SANTA FE
}

Eje 2: Tecnología para la construcción sustentable

Arq. Sebastián E. Puig ${ }^{1}$

Arq. Guillermo Aguirre ${ }^{2}$

Arq. Alberto Maidana ${ }^{3}$

Becario Leandro D. Fontanetto 4

\begin{abstract}
${ }^{1}$ Laboratorio de Técnicas y Materiales (LATMAT) - Instituto Regional de Estudios del Habitat (IREH). Facultad de Arquitectura, Diseño y Urbanismo - Universidad Nacional del Litoral, Santa Fe, Argentina, sebaspuig@gmail.com

2 Laboratorio de Técnicas y Materiales (LATMAT) - Instituto Regional de Estudios del Habitat (IREH). Facultad de Arquitectura, Diseño y Urbanismo - Universidad Nacional del Litoral, Santa Fe, Argentina, guillermomartinaguirre@gmail.com

${ }^{3}$ Laboratorio de Técnicas y Materiales (LATMAT) - Instituto Regional de Estudios del Habitat (IREH). Facultad de Arquitectura, Diseño y Urbanismo - Universidad Nacional del Litoral, Santa Fe, Argentina, amaidana@fadu.unl.edu.ar

${ }^{4}$ Laboratorio de Técnicas y Materiales (LATMAT) - Instituto Regional de Estudios del Habitat (IREH). Facultad de Arquitectura, Diseño y Urbanismo - Universidad Nacional del Litoral, Santa Fe, Argentina, lea_06_010@hotmail.com
\end{abstract}

\section{RESUMEN}

La escasa disponibilidad energética en la Argentina y el elevado consumo, es una problemática que viene incrementándose, cuando se analiza principalmente el año 2015 , año en que se declara la emergencia energética según la información pública disponible.

En este contexto, según lo señala el informe anual elaborado por el Ministerio de Energía y Minería de la Nación del año 2015 el conjunto de usuarios compuesto por el sector residencial, comercial y público consumen el $37 \%$ de la energía de nuestro país, y el $72 \%$ de esa energía es producto del gas distribuido en redes y gas natural licuado (GNL). En invierno la calefacción representa el $51 \%$ del consumo de gas lo que representa un problema que como arquitectos debemos afrontar.

Desde el punto de vista medioambiental, social y económico; y también político sería conveniente subsidiar mejoras en la eficiencia térmica y en la utilización de energías renovables antes que continuar subsidiando la energía. 
El presente trabajo de investigación es resultado de indagaciones en la temática, formuladas en ámbito del proyecto de investigación denominado "Arquitectura Sustentable, desarrollo experimental de un módulo habitacional con consumo de energía "0", bajo la dirección del Arq. Alberto Maidana, del cual participamos como investigadores. El mismo se desarrolla en el marco del Curso de Acción para la Investigación y Desarrollo de la UNL, convocatoria 2016.

El propósito de este trabajo es estudiar la factibilidad de un sistema basado en colectores solares para la provisión de agua caliente sanitaria (ACS) para el ahorro energético de una de las cien viviendas sociales de 2 dormitorios que se construyeron en el norte de la ciudad de Santa Fe, que no cuenta con gas natural de red. Para realizar el trabajo se relevaron los datos climáticos, solares, la demanda térmica, como también la disponibilidad del lugar para el cálculo del área de los colectores según el proyecto ejecutivo. Para realizar las verificaciones de datos se utilizó el software de gestión de energías limpias Retscreen $®$, de origen canadiense. El mismo permite la identificación exhaustiva, la evaluación y la optimización de la viabilidad técnica y financiera de proyectos potenciales de energía renovable y de eficiencia energética. Los resultados que se obtuvieron determinaron la viabilidad de la inversión inicial, disminuyendo el consumo energético de energías convencionales y reduciendo las emisiones de gases de efecto invernadero $\mathrm{CO}_{2}$.

\section{PALABRAS CLAVES: CLIMATIZACIÓN - COLECTORES SOLARES PLANOS - AHORRO DE ENERGÍA}

\section{INTRODUCCIÓN}

La escasa disponibilidad, el costo y el elevado consumo de la energía en la Argentina, es una problemática que viene incrementándose, cuando se analiza principalmente el año 2015, año en que se declara la emergencia energética (Telam, 2015)

Los gastos de importación de gas natural licuado y combustibles llegaron a 26.640 millones de dólares. En la actualidad, el gobierno nacional busca reducir al 50\% las compras de gas licuado con yacimientos no convencionales en Vaca Muerta. Esto no representa una baja en el consumo de energía, lo que permite inferir que la estrategia actual es reducir costos buscando disminuir su importación para lo cual se utilizan recursos propios que se están comenzando a explotar (Spaltro, 2017). Ahora bien, esta situación en sí misma no enfrenta el problema central que actualmente nos está afectando, es decir, el consumo excesivo de energía que permite llegar a niveles de confort necesarios en las edificaciones, problema al que los diseñadores del hábitat tienen que dar respuesta.

Desde el punto de vista medioambiental, social, económico y también político sería conveniente subsidiar mejoras en relación al uso de energías renovables antes que continuar subsidiando un consumo poco controlado de la energía.

En este contexto el gobierno provincial de Santa Fe, entregó un complejo habitacional de 100 viviendas, correspondiente al Plan de Viviendas de Emergencia Hídrica de 2003.

Las viviendas se edificaron con el prototipo "vivienda evolutiva" (V.E.), utilizando un modelo tradicional de construcción. Cada unidad tiene 55 metros cuadrados cubiertos y cuenta con dos dormitorios, cocina, baño y comedor. 
El propósito de este trabajo es estudiar la factibilidad de un sistema basado en colectores solares para la provisión de agua caliente sanitaria (ACS) para el ahorro energético de una de las cien viviendas sociales de 2 dormitorios que se construyeron en el norte de la ciudad de Santa Fe, que no cuenta con gas natural de red.

\section{DESARROLLO}

\section{UBICACIÓN Y CARACTERÍSTICAS DEL PROYECTO}

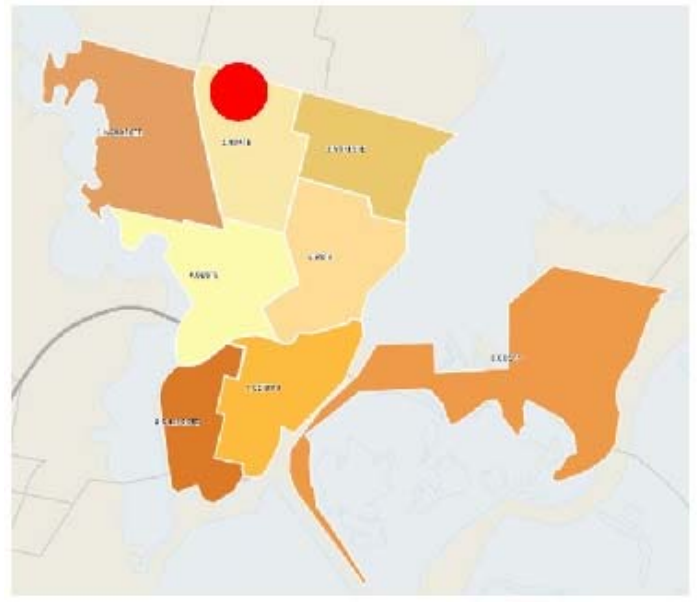

Fig. 1. Mapa Geográfico de la Ciudad de Santa Fe. Ubicación del conjunto de viviendas.

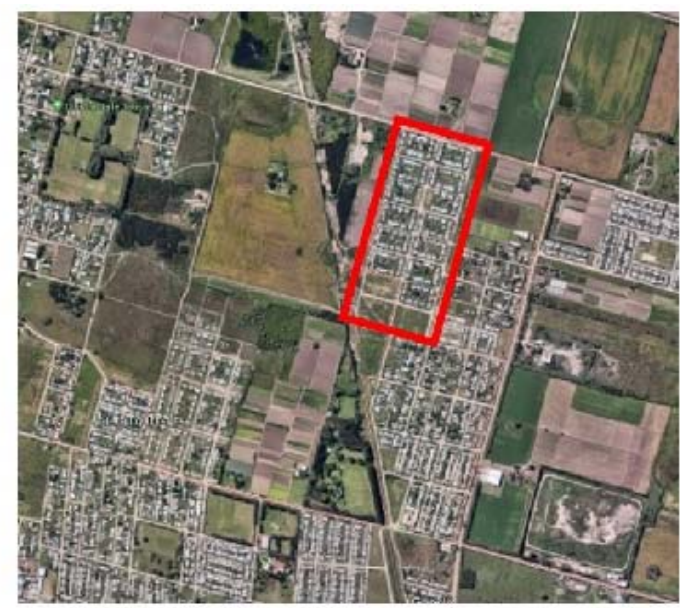

Fig. 2. Lugar de emplazamiento de las 100 viviendas de 2 dormitorios gestionadas por la DPVyU de la Provincia de Santa Fe

La ciudad de Santa Fe, se ubica en las coordenadas $31^{\circ} 37^{\prime} 59^{\prime \prime}$ latitud Sur y $60^{\circ} 42^{\prime} 00^{\prime \prime}$ longitud Oeste situada en el valle de los ríos Paraná y Salado, con una altitud de 21 m.s.n.m. El Clima de la ciudad de Santa Fe recibe una marcada influencia del río Paraná en las condiciones climáticas, atenuando sus características de mediterraneidad, es por esto que se define como como subtropical húmedo, promediando los $977 \mathrm{~mm}$ de precipitaciones. Las temperaturas oscilan entre los $7^{\circ} \mathrm{C}$ y los $17.8^{\circ} \mathrm{C}$ en invierno y entre $19.5^{\circ} \mathrm{C}$ y $32.1^{\circ} \mathrm{C}$ en verano.

La vivienda se ubica en el ejido urbano, su entorno comprende viviendas cercanas de baja altura, un centro recreativo (club) y parcelas sin construcciones. El prototipo consta de una planta baja en el cual se emplazan dos dormitorios, una cocina comedor, un núcleo sanitario comprendido por cocina y lavadero.

Para el sistema de agua caliente sanitaria (ACS) por colectores solares planos se tuvo en cuenta la radiación del sitio y el espacio físico disponible, relevando los datos in situ de dimensionamiento de la superficie de techo disponible de la vivienda. 


\section{UBICACIÓN DE LA VIVIENDA EVOLUTIVA Y SECTOR DONDE SE INSTALARÁN LOS COLECTORES.}

A continuación, vista de la vivienda evolutiva (V.E.) y el sector del sitio elegido donde se ubicarán los colectores solares.

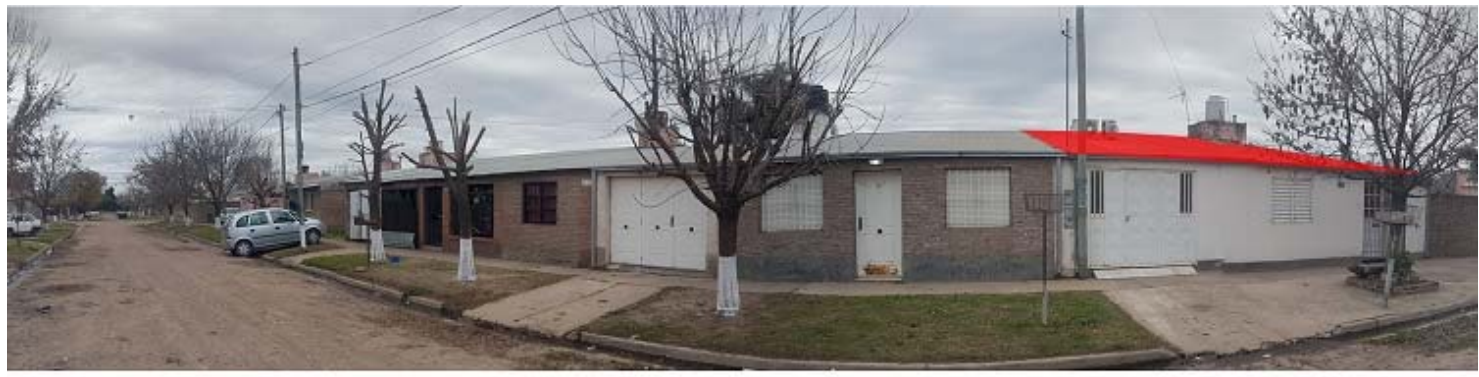

Fig. 3. Sitio de Ubicación de los colectores solares planos

\section{EVALACIÓN DEL RECURSO}

\section{DESCRIPCIÓN DEL SISTEMA ACTUAL DE AGUA CALIENTE SANITARIA (A.C.S.)}

El sistema de calentador de ACS actual funciona los 365 días del año. Según el uso de la vivienda, hay tres momentos en el día en que la demanda de ACS se eleva. A la mañana temprano, al mediodía y en el horario vespertino.

La distribución de agua caliente se realiza través de tuberías interiores conectadas a un Termotanque eléctrico LONGVIE de 60lts.

Sus características son las siguientes:

Temperaturas del sistema del termotanque:

$\mathrm{T}^{\circ}$ arranque (entrada) $=16{ }^{\circ} \mathrm{C}-23^{\circ} \mathrm{C}$

$\mathrm{T}^{\circ}$ normal de funcionamiento $($ salida $)=48{ }^{\circ} \mathrm{C}$

$\mathrm{T}^{\circ}$ Máxima del equipo (límite) $=70^{\circ} \mathrm{C}$

\section{ESTIMACIÓN DE LAS NECESIDADES DE ENERGÍA}

Consumo actual de energía

Para determinar la energía utilizada por la vivienda, en un promedio anual, se accede al calculador de la Empresa provincial de energía de Santa Fe (EPESF).

Energía utilizada: convencional. Tipo: Eléctrica; Consumo Anual de Energía $=3654 \mathrm{Kw} / \mathrm{año}=$ $3,654 \mathrm{Mw} / \mathrm{año}$

\section{DIMENSIONAMIENTO DEL EQUIPO DE AGUA CALIENTE SOLAR}

El equipo de agua caliente por energía solar debe tener una capacidad, de modo que, en la hora pico de consumo, disponga de la cantidad de agua necesaria a la temperatura requerida.

El cálculo se divide en dos partes fundamentales: 
- Dimensionamiento del colector solar

- Capacidad del tanque de almacenamiento

a. Consumo de Agua según la actividad:

\begin{tabular}{lll} 
Criterio de consumo & Litros/día a $\mathbf{4 5} \mathbf{C}$ & \\
\hline Viviendas unifamiliares & $50-60$ & por persona \\
Viviendas multifamiliares & 30 & por persona \\
Hospitales y clínicas & 80 & por cama \\
Hoteles **** & 100 & por cama \\
Hoteles *** & 80 & por cama \\
Hoteles/Hostales ** & 60 & por cama \\
Camping & 60 & por emplazamiento \\
Hostales/Pensiones * & 50 & por cama \\
Residencias (ancianos, estudiantes, etc.) & 80 & por cama \\
Vestuarios/Duchas colectivas & 20 & por servicio \\
Escuelas & 5 & por alumno
\end{tabular}

Tabla 1. Consumo de Agua Caliente Sanitaria según la actividad.

\section{b. Dimensionamiento del colector solar}

El colector solar se calcula con la siguiente fórmula:

$$
S=\frac{C d \cdot(t s-t e)}{I t . \eta}=\left(m^{2}\right)
$$

\section{Donde:}

$\mathbf{C d}=$ Caudal diario de agua necesaria a calentar. $($ Ver Tabla 1$)$

Determinamos el caudal diario necesario para los 4 ocupantes de la vivienda.

4 personas $\times 60$ [litros/persona/día] = 240 litros $/$ día $=\mathbf{C d}$

Ts $=$ Temperatura de salida del agua del tanque $\left({ }^{\circ} \mathrm{C}\right)$. Adoptamos $45^{\circ} \mathrm{C}$. (Temperatura de confort del agua caliente, constante de diseño)

$\mathrm{Te}=$ Temperatura de Entrada del agua de la red al tanque $\left({ }^{\circ} \mathrm{C}\right)$ Adoptamos $16,2^{\circ} \mathrm{C}$. (Es el agua que viene de la red, o del tanque de reserva, normalmente oscila entre $16,2^{\circ} \mathrm{C}$ a $21^{\circ} \mathrm{C}$ )

It = Intensidad de radiación solar diaria sobre el plano inclinado del colector $\left(\mathrm{kcal} / \mathrm{día} \mathrm{m}^{2}\right)$. En Santa $\mathrm{Fe}$, según los datos brindados por el Informe técnico de avance del ESTADO DE LA RED SOLARIMÉTRICA DE LA PROVINCIA DE SANTA FE de junio del 2016 (Ver Ref. [23]), para nuestra zona vamos a tener $\mathbf{4 0 4 1} \mathrm{kcal} / \mathrm{dí}^{\mathrm{a}} \mathrm{m}^{2}$

$\boldsymbol{\eta}=$ Rendimiento del colector heat pipe de tubos evacuados (\%), para la marca SUNGREEN el rendimiento es del $\mathbf{9 4 \%}$ de eficiencia.

$$
S=\frac{C d \cdot(\text { ts }-t e)}{I t . \eta}=\frac{240 l t s \cdot\left(45^{\circ} C-16,2^{\circ} C\right)}{4041 \mathrm{kcal} / \text { día }^{\circ} 2 \cdot 0,94}=1.82 \mathrm{~m} 2
$$


El área necesaria para nuestro colector es de 1,82 $\mathrm{m2}$. Los colectores de la firma SUNGREEN SRL tienen una dimensión de 2,10 x 1,80 m, donde su área de captación neta es de 3,30m2 por lo cual se utilizaría 1 colector.

\section{ESPECIFICACIONES TÉCNICAS DEL COLECTOR}

- Colector de Tubos de Vacío - Sistema Heat Pipe

- Material del Heat Pipe - Aleta de Aluminio 0.25mm de espesor

- Absortancia Solar $\alpha-94 \%$

- Emisividad Solar $\varepsilon-4 \%$

- Rendimiento del colector $=0,94$

- Presión Máxima de Diseño - 10 bares

- Caudal de diseño - 167 (Its/día)

- Superficie de Captación Bruta - 3,60 m2

- Superficie de Captación Neta - 3,30 m2

- Marco - Aleación de aluminio - espesor $1.2 \mathrm{~mm}$

- Material de Tubo Cabezal - Cobre TP2 de alta pureza

- Aislación del Cabezal - Doble: espuma de poliuretano y silicato de aluminio rodeando la tubería principal

- Peso Total: Aprox. $113 \mathrm{~kg}$ (con agua)

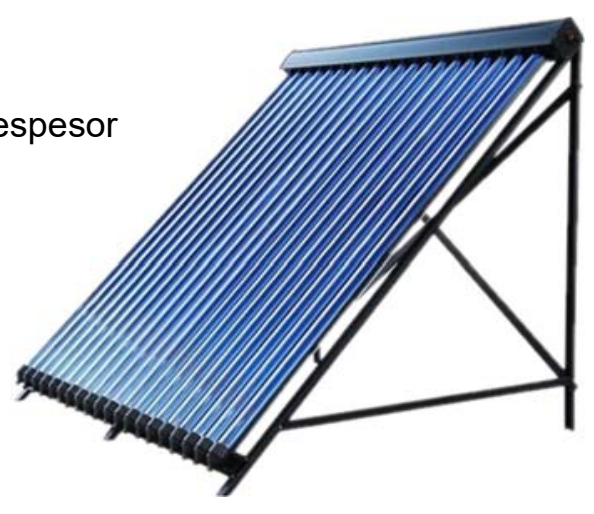

Fig. 4 Modelo CTVJ-20 de la marca SUNGREEN S.R.L.

\section{a- Dimensionamiento del tanque térmico de almacenamiento}

Considerando que el consumo necesario es de 60 its por persona el tanque de almacenamiento será de 60 Its $\times 4$ personas $=240$ Its.

Los Tanques térmicos de acumulación de 1 serpentín de la firma SUNGREEN Santa Fe tienen una capacidad de 300Its, para lo cual necesitaríamos 1 tanque térmico.

\section{ESPECIFICACIONES TÉCNICAS DEL TANQUE TÉRMICO}

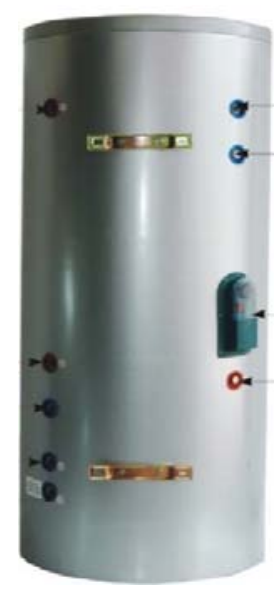

- Capacidad: 300lts

- - Aislación: Poliuretano / e: $45 \mathrm{~mm}$

- - Serpentín (Solar): d: $16 \mathrm{~mm}$ x L: $14 \mathrm{~m}$

- - Dimensiones Generales: d: $580 \mathrm{~mm}$ x h: $1700 \mathrm{~mm}$

Fig. 5 Modelo TP1J de la marca SUNGREEN Santa

- Peso Neto: $65 \mathrm{~kg}$

- $\quad$ - Presión de Trabajo: 4 bar

- - Vida Util: 20 años

\section{b- Posición del colector:}

\section{Irradiancia del sitio}

La Irradiancia Solar establece la variable más importante en la utilización de este recurso que representa la potencia energética recibida por unidad de superficie, para calcular el área de captación de colectores solares, es decir el recurso energético a partir del cual se dimensionará el sistema de aprovechamiento solar térmico de agua caliente. Se estimó la Irradiancia Solar del sitio de emplazamiento del proyecto, se han empleado los datos publicados por la NASA (Tabla 2) para 
las coordenadas geográficas correspondientes a la localidad de Santa Fe, Latitud: -31.63 / Longitud: -60.7 .

\begin{tabular}{|l|c|c|c|c|c|c|c|c|c|c|c|c|c|}
\hline \multicolumn{10}{|c|}{ Máxima Radiación Incidente sobre un punto de la superficie inclinada del Ecuador (kWh/m2/día) } \\
\hline PARAMETROS & Ene & Feb & Mar & Abr & May & Jun & Jul & Ago & Sep & Oct & Nov & Dic & Prom. \\
\hline $\begin{array}{l}\text { SI_EF_TILTED_ } \\
\text { SURFACE_0 }\end{array}$ & 6.83 & 6.03 & 4.96 & 3.75 & 2.94 & 2.43 & 2.77 & 3.59 & 4.89 & 5.51 & 6.57 & 6.91 & 4.76 \\
\hline $\begin{array}{l}\text { SI_EF_TILTED_ } \\
\text { SURFACE_16 }\end{array}$ & 6.65 & 6.09 & 5.29 & 4.27 & 3.59 & 3.07 & 3.47 & 4.25 & 5.29 & 5.68 & 6.46 & 6.68 & 5.07 \\
\hline $\begin{array}{l}\text { SI_EF_TILTED_ } \\
\text { SURFACE_31 }\end{array}$ & 6.14 & 5.82 & 5.32 & 4.53 & 4.00 & 3.50 & 3.93 & 4.62 & 5.45 & 5.54 & 6.01 & 6.11 & 5.07 \\
\hline $\begin{array}{l}\text { SI_EF_TILTED_ } \\
\text { SURFACE_46 }\end{array}$ & 5.38 & 5.29 & 5.06 & 4.54 & 4.18 & 3.72 & 4.17 & 4.73 & 5.32 & 5.12 & 5.32 & 5.32 & 4.85 \\
\hline $\begin{array}{l}\text { SI_EF_TILTED_ } \\
\text { SURFACE_90 }\end{array}$ & 2.18 & 2.50 & 2.93 & 3.23 & 3.36 & 3.17 & 3.49 & 3.57 & 3.39 & 2.59 & 2.21 & 2.12 & 2.89 \\
\hline SI_EF_OPTIMAL & 6.83 & 2.11 & 5.34 & 4.56 & 4.19 & 3.76 & 4.20 & 4.73 & 5.46 & 5.68 & 6.58 & 6.91 & 5.36 \\
\hline $\begin{array}{l}\text { SI_EF_OPTIMAL } \\
\text { ANG }\end{array}$ & -1 & -11 & -25 & -39 & -50 & -54 & -53 & -45 & -32 & -16 & -3 & -0 & -27 \\
\hline $\begin{array}{l}\text { SI_EF_TILTED_ } \\
\text { ANG_ORT }\end{array}$ & $\mathrm{N}$ & $\mathrm{N}$ & $\mathrm{N}$ & $\mathrm{N}$ & $\mathrm{N}$ & $\mathrm{N}$ & $\mathrm{N}$ & $\mathrm{N}$ & $\mathrm{N}$ & $\mathrm{N}$ & $\mathrm{N}$ & $\mathrm{N}$ & $\mathrm{N}$ \\
\hline
\end{tabular}

Tabla 2: Irradiancia solar máxima mensual. Fuente: NASA Solar Irrandiance for Equator Facing Tilted Surfaces (Set or Surfaces)

En la tabla 2 se destaca el mes de junio de mayor demanda de energía para calentar el agua para uso sanitario y el ángulo de inclinación y orientación óptimo para el mes más desfavorable. 


\section{ANÁLISIS A TRAVÉS DEL SOFTWARE RETSCREEN®.}

Se destaca que los métodos de cálculo no se basan en valores instantáneos, hay variables que no permiten predecir el comportamiento del sistema. Lo que se obtendrá son estimaciones de su comportamiento bajo condiciones medias mensuales.

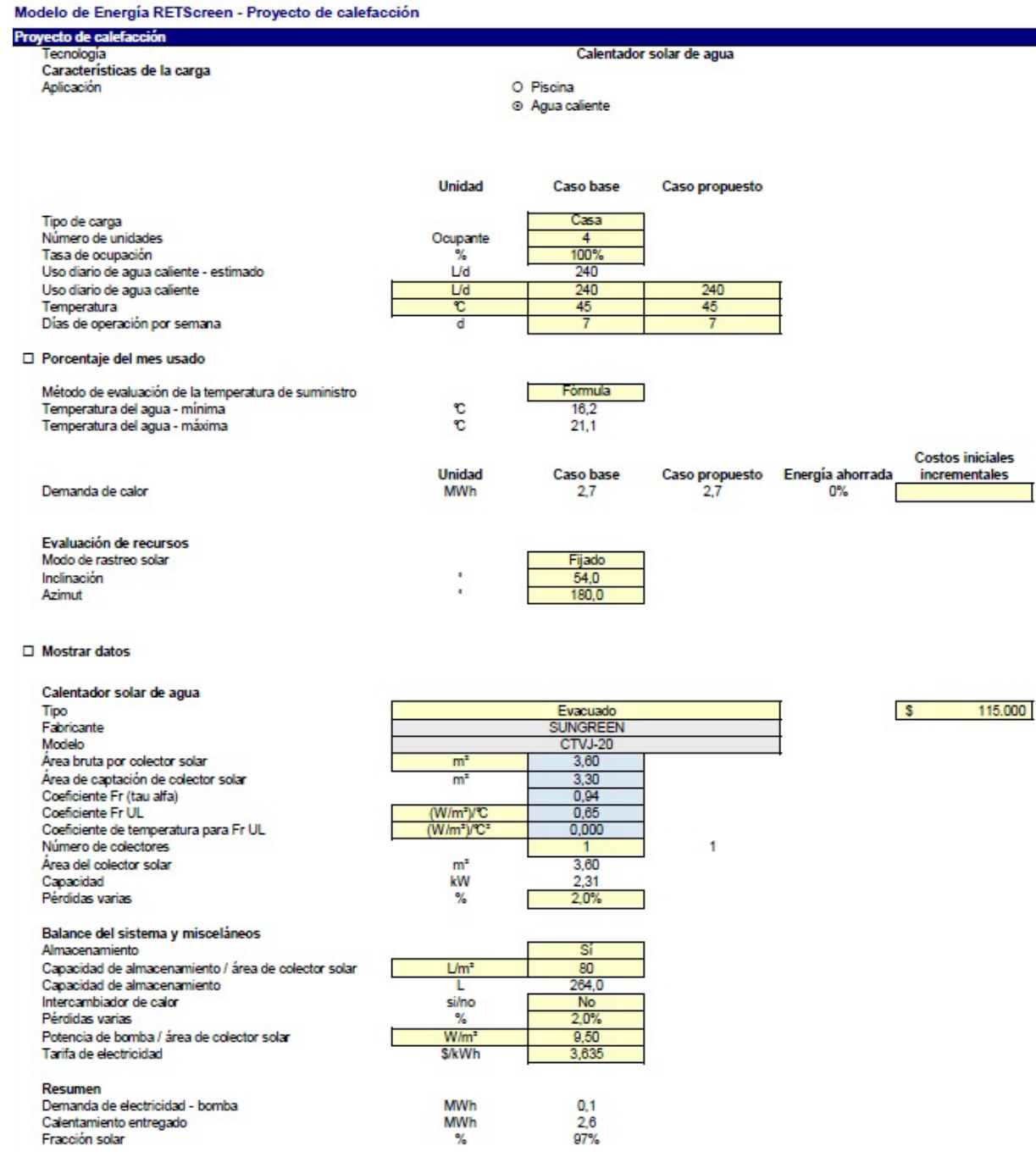

Dada estas pautas, es que se utilizaran fuentes auxiliares de energía que cubrir el déficit bajo condiciones climáticas adversas $o$, cuando se haya previsto dimensionar la fracción de la demanda no cubierta por la energía solar.

Para el cálculo de la fracción solar se adoptan los colectores solares según las especificaciones técnicas provistas por el fabricante. Para el caso de estudio, utilizamos los datos brindados por la empresa SUNGREEN Santa Fe.

Fig. 6 Resultados arrojados por el software de gestión de energías limpias RETSCREEN®
Fracción Solar promedio obtenida: 97\%, logrando cubrir la demanda energética actual. 


\section{ESQUEMA DEL SISTEMA DE COLECTOR PROPUESTO}

En la figura se representa simbólicamente el sistema de ACS propuesto. El mismo funciona de forma combinada con el termotanque eléctrico, en donde el controlar digital es el que hace funcionar el circuito primario de agua cuando aumenta la demanda de ACS. De esta manera el sistema ahorra energía convencional (electricidad) cuando existe un aporte de energía solar térmica de los colectores. Cuando el aporte es insuficiente, el termotanque eléctrico funciona de manera independiente. Para el caso de los sistemas Split, se puede prescindir del termotanque eléctrico dado que a esta clase de equipos se le puede adicionar una resistencia eléctrica.

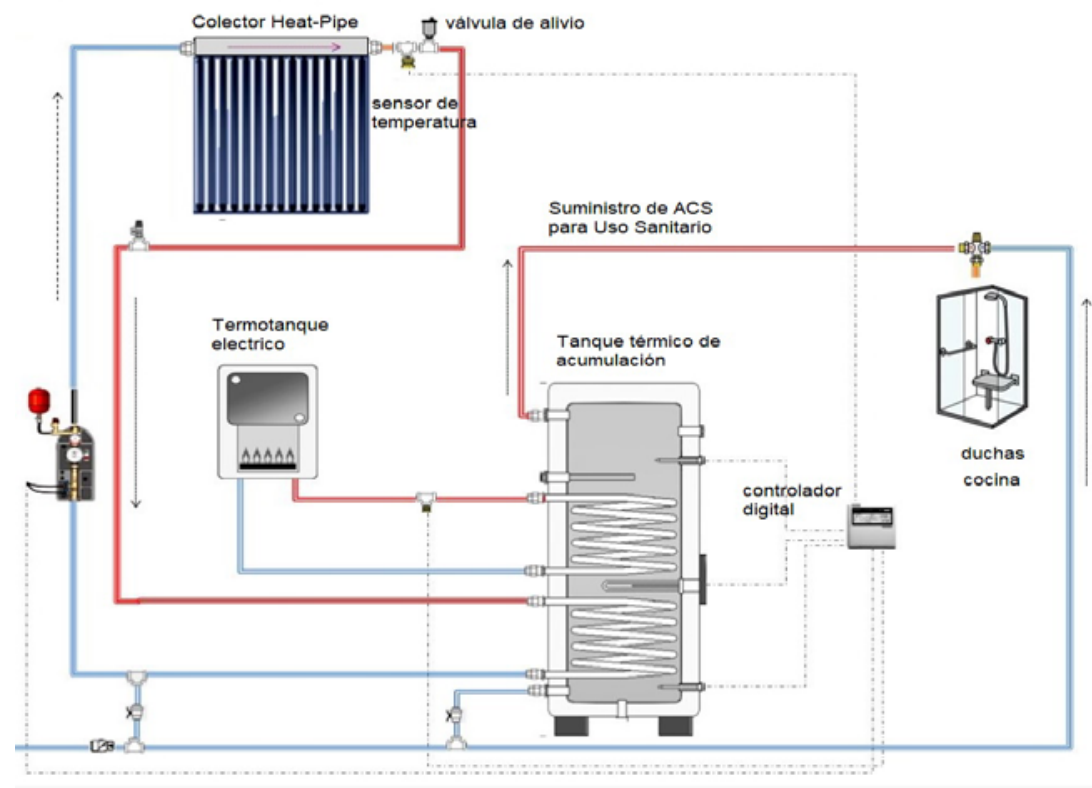

Fig. 7 Esquema gráfico propuesto del sistema

\section{CÁLCULO DE COSTOS Y ANALISIS FINANCIERO DEL SISTEMA}

En la tabla 3 se visualiza los costos generales del proyecto y luego a través del software Retscreen ${ }^{\circledR}$ obtenemos el balance económico (retorno del capital).

\begin{tabular}{|l|c|cr|c|}
\hline \multicolumn{1}{|c|}{ COMPONENTES } & Cantidad & Precio Unitario & Precio Total \\
\hline Colector Heat pipe de 20 tubos & 1,0 & $\$ 22.061,00$ & $\$ 22.061,00$ \\
\hline $\begin{array}{l}\text { Tanque Térmico 1 serpentina } \\
\text { 200lts }\end{array}$ & 1,0 & $\$$ & $24.049,62$ & $\$ 24.049,62$ \\
\hline Componentes del Sistema Split & 1,0 & $\$$ & $21.000,00$ & $\$ 24.950,00$ \\
\hline \multicolumn{4}{|c|}{ COSTO DEL EQUIPO } & $\$ 71.060,62$ \\
\hline MATERIALES PARA INSTALACION & $\$ 26.174,23$ \\
\hline \multicolumn{3}{|c|}{ COSTO DE LA INSTALACION (20\% C.T. COMP) } & $\$ 17.765,16$ \\
\hline \multicolumn{4}{|c}{ COSTO TOTAL } & $\$ 115.000,01$ \\
\hline
\end{tabular}

En cuanto

Tablas 3 Costos de componentes, materiales e instalación

al análisis financiero la vivienda posee un gasto actual de $\$ 13.944$ anuales por consumo de energía eléctrica según las estimaciones que se realizaron con el calculador de la EPESF, en nuestro caso de estudio introducimos una inflación del $30 \%$ anual para nuestro país. La instalación del sistema 
Split representa un ahorro del $97 \%$ de la demanda anual. Luego de 8,6 años estaremos recuperando la inversión y el proyecto comienza a tener ganancia económica por ahorro en el consumo energético.

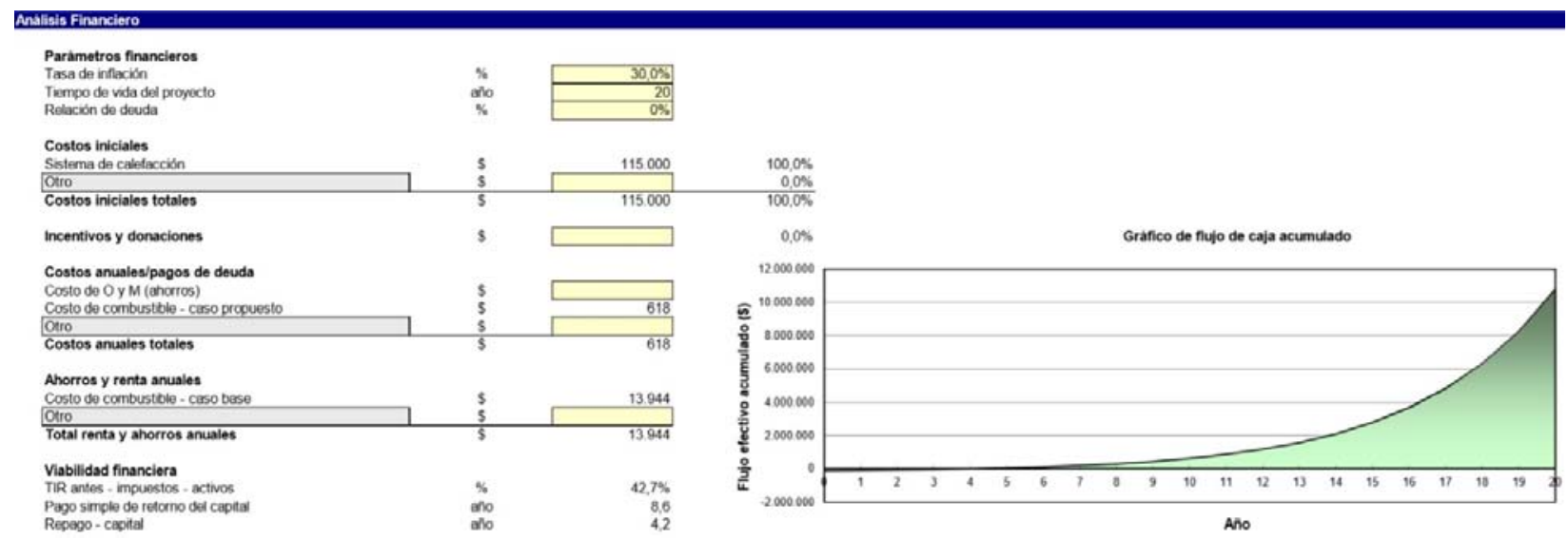

Fig. 8 Resultados del análisis financiero del software Retscreen®

\section{CÁLCULO DE GASES DE EFECTO INVERNADERO.}

Si consideramos que para generar $1 \mathrm{kwh}$ de energía eléctrica con combustibles no renovables, emitimos $0,385 \mathrm{~kg}$ de $\mathrm{CO}_{2}$; entonces si la Vivienda, consume anualmente $3654 \mathrm{kwh}$ equivale a $1406,79 \mathrm{Kg}$ de $\mathrm{CO}_{2}$ por año.

Ahorro de emisiones del proyecto en el período de retorno del capital (8,6 años); $1406,79 \mathrm{~kg}$ de $\mathrm{CO}_{2} \times 8$ años $=12098,35 \mathrm{~kg}$ de $\mathrm{CO}_{2}$.

Ahorro de emisiones del proyecto en el período de Vida Útil (20 años); $1406,79 \mathrm{~kg}$ de $\mathrm{CO}_{2} \times 20$ años $=28135,8 \mathrm{~kg}$ de $\mathrm{CO}_{2}$.

\section{CONCLUSIONES}

Del análisis del proyecto que se presentó, se concluye que el sistema de colector heat pipe es un aporte fundamental para el ahorro energético en el uso del ACS de la Vivienda Evolutiva.

Los resultados que se obtuvieron determinaron la viabilidad de la inversión inicial, disminuyendo el consumo energético de energías convencionales y reduciendo las emisiones de gases de efecto invernadero $\mathrm{CO}_{2}$. No obstante queda a futuro, estudiar las condiciones energéticas de la vivienda en cuanto a su construcción edilicia, evaluando la posibilidad de incorporar mejoras a la construcción de manera de poder hacerla más eficiente.

\section{BIBLIOGRAFÍA}

Departamento de Agricultura, Ganadería y Medio ambiente del Gobierno de Aragón, Cámara de Comercio e Industria de Zaragoza. Cálculo automático de emisiones totales en relación a los consumos energéticos de sus instalaciones. Recuperado: https://www.camarazaragoza.com/wpcontent/uploads/2012/10/calculoemisiones.xls 
Porfiri F, Bouhier A y Lara M (2013). ASADES. Sistema de ahorro energético basado en colectores planos para la calefacción central de una escuela de la localidad de Alcorta.

Puig S; Material de Cátedra de Energía Solar Térmica. Instalaciones I (FADU-UNL). Laboratorio de Técnicas y Materiales (LATMAT). Dirección Dra. Arq. Ma. Laura Tarchini; Coordinador Técnico Esp. Arq. Rodolfo Bellot.

Retscreen® software, licencia gratuita. Recuperado; www.nrcan.gc.ca/energy/software-tools/7465

Spaltro, S. El Gobierno apuesta a reducir 50\% las compras de gas licuado en 2 años. El Cronista [en línea]. Buenos Aires, 18 de setiembre 2017. Disponible en: $<$ https://www.cronista.com/economiapolitica/El-Gobierno-apuesta-a-reducir-50-las-compras-degas-licuado-en-2-anos-20170918-0028.html>. [Acceso: 20 marzo 2018].

Secretaría de Estado de la Energía de la Provincia, a través de la Subsecretaría de Energías Renovables (SSER). (2016). Informe Técnico de Avance, ESTADO DE LA RED SOLARIMÉTRICA DE LA PROVINCIA DE SANTA FE. Recuperado:

https://www.santafe.gov.ar/noticias/recursos/.../10/2015-10-20NID_218644O_1.pdf

SUNGREEN Santa Fe, www.sgenergia.com.ar (año 2018). Consulta de presupuesto de equipos e instalación. [Acceso: 18 febrero 2018]

Telam. El gobierno decreto la emergencia energética todo el país diciembre de 2017. Infobae [en línea]. Buenos Aires 15 de setiembre 2015. Disponible en:

$<$ https://www.infobae.com/2015/12/15/1776772-el-gobierno-decreto-la-emergencia-energeticatodo-el-pais-diciembre-2017/>. [Acceso: 22 febrero 2018]. 